Revue d'histoire de l'Amérique française

REVUE D.HISTOIRE DE L'AMÉRIQUE FRANÇAISE

\title{
Les Gaultier de la Veranderie en France et au Canada et leurs relations par delà l'océan (suite et fin)
}

\section{Antonio Champagne}

Volume 13, numéro 1, juin 1959

URI : https://id.erudit.org/iderudit/301959ar

DOI : https://doi.org/10.7202/301959ar

Aller au sommaire du numéro

Éditeur(s)

Institut d'histoire de l'Amérique française

ISSN

0035-2357 (imprimé)

1492-1383 (numérique)

Découvrir la revue

Citer ce document

Champagne, A. (1959). Les Gaultier de la Veranderie en France et au Canada et leurs relations par delà l'océan (suite et fin). Revue d'histoire de l'Amérique française, 13(1), 97-122. https://doi.org/10.7202/301959ar d'utilisation que vous pouvez consulter en ligne. 


\title{
DOCUMENTS INÉDITS
}

\author{
LES GAULTIER DE LA VERANDERIE EN FRANCE ET AU CANADA \\ ET LEURS RELATIONS PAR DELÀ L'OCEAN
}

(Documents et commentaires)

(suite)*

V. LA VÉRENDRYE PASSE EN FRANCE À SON TOUR: 1707-1712. DÉMARCHES POUR Y RETOURNER EN 1724-1727.

Ce titre nous amène à une période plutôt incertaine et obscure de l'histoire de La Vérendrye, celle qui s'écoule entre son départ pour la France en 1707 et son retour au Canada en 1712. Mais l'incertitude même dans laquelle on se débattait jusqu'ici ajoutera à l'intérêt de cette étude.

D'après ce qui précède, La Vérendrye n'a évidemment pas servi sous son frère ou à côté de lui, comme certains auteurs ont écrit. C'est au contraire la nouvelle de la mort de Louis qui, selon toutes les apparences, a déclenché la chaîne des faits suivants : 1707 ;

1. Partage de Marie Boucher entre ses enfants: 1er juillet drye ;

2. Pierre Gaultier de Boumois prend le nom de La VérenFrance;

3. Décision de La Vérendrye d'aller servir dans l'armée de

4. La Vérendrye vend à Christophe Dufrost de La Jemmeraye sa part de la Grande Ile de Varennes: 27 octobre 1707;

5. Contrat de mariage de La Vérendrye avec Marie-Anne Dandonneau : 9 novembre 1707.

6. Départ de La Vérendrye pour la France.

Nous ne savons pas à quel moment de cet été La Vérendrye se décida à partir.

* Voir notre revue, XII: 262-277, 411-427; 574-581.

N.B. On voudra bien corriger ainsi notre précédent article: a) 7e parag. lire tous héritiers. b) p. 579 , 6e 1., lire quelque chose. c) p. 581, compléter la dernière phrase: $A$ part la vague mention de 1703, nous n'entendrons plus parler de lui... 
Le premier document que nous connaissons à ce sujet est l'autorisation que lui donne Pierre Boucher, son grand-père et subrogé-tuteur, de vendre pour 800 livres sa moitié de la Grande Ile de Varennes, afin de se procurer des fonds pour le voyage. Le but de La Vérendrye, tel qu'indiqué par Pierre Boucher, est «de trouver de l'emploi et de s'avancer, ce qu'il ne pourrait faire en restant en ce pays ». Le document, sous seing privé, est signé de Pierre Boucher et daté de «Boucharville (sic) 27 octobre $1707 \gg .^{33}$

Le même jour, devant Pierre Raimbault, avait lieu la vente de la terre ci-dessus, par La Vérendrye, à son beau-frère Christophe Dufrost de La Jemmeraye.

Voici ce document dans ses parties essentielles:

Pièce No $14 a$. No 1362.

27 octobre 1707. Vente par Pierre Gaultier à Christophe Dufro.

Pardevant..., fut présent Pierre Gaultier, écuyer, sieur de Boumois et de la Veranderie, lequel, assisté de dame Marie Boucher, sa mère et tutrice, et aussi de l'avis et consentement du sieur Pierre Boucher, écuyer, seigneur de Boucharville (sic), son grand-père et subrogé-tuteur...,

désirant passer en l'ancienne France pour s'y établir plus avantageusement qu'en ce pays, .... ce qu'il ne peut exécuter sans aliéner une partie de ses biens,

a reconnu et confessé avoir vendu et délaissé à Christophe Dufro, écuyer, sieur Delagemeraye, enseigne de vaisseau et capitaine d'une compagnie du détachement de la marine, acquéreur,

la moitié de la Grande Ile de Varennes, à prendre par le bout d'en bas, tenant l'autre bout à l'autre moitié de ladite île, appartenant à la demoiselle de la Veranderie, sa sœur, sans en rien excepter que la jouissance pour ladite dame sa mère sa vie durant, ainsi que le tout est stipulé au contrat de partage que ladite dame a fait de ses biens le premier juillet dernier, ladite moitié d'île faisant partie du fief de Varennes....;

cette vente faite moyennant la somme de 800 livres, laquelle le sieur vendeur a reconnu avoir aujourd'hui reçue du sieur de la Gemeraye,

et en outre à la charge de pouvoir par le vendeur exercer la faculté de reméré et retirer ladite moitié d'île lorsqu'il aura atteint sa majorité, en sorte que ladite faculté sera nulle un an après ladite majorité, ou, s'il repasse en ce pays, un an seulement après qu'il $\mathrm{y}$ sera de retour; passé lequel temps, en rendant et payant par le sieur vendeur au sieur acquéreur pareille somme de 800 livres;

quoi faisant, le sieur vendeur rentrera en la propriété de ladite moitié d'île vendue...

33 L'original appartient à la collection Montarville Boucher de la Bruère, laquelle est conservée aux archives de l'évêché, aux Trois-Rivières.

Une reproduction photographique en a été donnée dans le Catalogue de l'Exposition rétrospective des Colonies françaises de l'Amérique du Nord tenue à Paris en avril-juin 1929, face à la page 63. - Voir aussi l'article de Montarville Boucher de la Bruère: «Pierre Boucher colonisateur 》, dans les Cahiers des Dix, (no 3) : 173-174. 
a été convenu que si, par quelque cause imprévue, le partage que ladite dame a fait venait à être changé, ladite moitié d'île présentement vendue ne se trouvant pas dans le lot du sieur vendeur, il sera tenu de fournir au sieur acquéreur d'autres terres de son lot, de même valeur que ladite moitié d'île.

Pour sûreté de ladite somme de 800 livres ladite dame s'est rendue plaige et caution pour le sieur de la Veranderie envers le sieur de La Gemeraye..., sous l'obligation et hypothèque de tous ses biens présents et à venir.

Même (elle) a, à ladite garantie, spécialement affecté l'usufruit qu'elle a sur toutes les terres et revenus de ladite seigneurie de Varennes...

Et pour l'exécution, ladite dame et le sieur de Boumois de la Veranderie a élu son domicile en la maison de ladite dame sise rue Saint-Vincent audit Ville-Marie, et le sieur acquéreur sa maison de demeure sise à Boucharville sur ses terres, et en cette ville la maison du sieur Ignace Gamelin sise rue Saint-Paul...

Fait et passé à Ville-Marie, étude dudit notaire, l'an 1707, le 27e jour d'octobre, (en) présence de sieurs Dominique Thaumur, chirurgien, et Jean Brunet, boucher.

(Signé): Marie Boucher veuve de Varenne, - P. Boumois, - Lagemerais, - Jean Brunet, - D. Thaumur.

P. Raimbault.

Pièce $N^{\circ} 14 b$.

(Annexe au précédent document, 17 août 1713).

Aujourd'hui, pardevant le notaire susdit, fut présent ledit sieur Boumois de la Veranderie, (lequel) a ratifié et confirmé la vente et paiement ci-dessus, et à la garantir s'est obligé... midi,

Fait et passé, étude dudit notaire, l'an 1713 , le $17 \mathrm{e}$ jour d'août avant

Et a signé: P. Gaultier de Laverandrye.

Pièce No $14 c$.

(Relevé par Aegidius Fauteux. Notes conservées à la Bibliothèque municipale de Montréal. Documents du régime français, liasse 57. - Ce document manque au greffe de Tailhandier).

Tailhandier, 2 novembre 1707. No 2271.

Procuration de Madeleine Gaultier, épouse Livilliers, Jacques-René Gaultier de Varennes, Jean-Baptiste Gaultier de Varennes, Marie-Marguerite Gaultier, épouse Puigibault, Marie-Renée Gaultier, épouse La Jemmeraye, Marie Boucher, veuve de René Gaultier de Varennes, comme tutrice de Pierre Gaultier de Boumois, - héritiers de feu Charles Gaultier de la Verandrye, commandant pour le roi à Arlon;

à Jean-Claude Louet, de Québec.

Cette série de documents, s'échelonnant du 1er juillet au 9 novembre 1707 , nous suggère d'importantes remarques, qui éclaireront toute la situation de La Vérendrye à cette époque. voyons que:

Tout d'abord, dans l'acte de partage du 1er juillet, nous

a) Louis, à qui seraient revenus les premiers droits en qualité d'aîné, s'il eût été vivant, n'est pas nommé. 
b) Jacques-René devient seigneur de Varennes, avec toutes les prérogatives attachées à ce titre, et, par exception, garde son nom de Varennes, qui correspond à celui de la seigneurie.

c) Pierre, appelé jusqu'ici «Boumois », reçoit pour la première fois le titre officiel de «La Veranderie », qui était celui de Louis et qu'il emploiera désormais, tout en conservant parfois son premier titre, de même que celui de « de Varennes ».

Dans ce partage, "Pierre Gaultier de Boumois et de la Veranderie, absent », retenu sans doute par ses devoirs militaires, reçoit la plus grande partie de la seigneurie du Tremblay et la moitié d'en bas de la Grande Ile de Varennes, ainsi que des droits qu'il partagera avec ses frères et sœurs sur la seigneurie de La Gabelle ou Saut de La Veranderie, sur une maison à Montréal et sur le moulin de Varennes.

Cet acte est passé à Montréal devant le notaire Pierre Raimbault, dans la maison de la dame de Varennes.

Les deux documents du 27 octobre nous mettent au courant d'une importante circonstance précédant le départ de La Vérendrye.

Ayant besoin d'argent pour le voyage, il obtient de ses tuteurs, c'est-à-dire de sa mère et de son grand-père Pierre Boucher, l'autorisation de vendre pour 800 livres la part qu'il a reçue dans la Grande Ile de Varennes, laquelle est attenante à l'autre part reçue par sa sœur Marguerite, mariée depuis peu à Louis Hingue de Puigibault.

Pierre Boucher, alors âgé de 85 ans, n'a pu se rendre à Montréal, mais il lui a donné une attestation sous seing privé. Muni de celle-ci, il se présente avec sa mère devant le notaire Raimbault, et celui-ci rédige l'acte de vente.

Le 2 novembre 1707, Louis La Vérendrye n'est pas mentionné parmi les héritiers possibles de Charles Gaultier de la Veranderie, ce qui confirme ce que nous avons dit de la date de sa mort (Pièce $\mathrm{N}^{\circ} 14 \mathrm{c}$ ).

Cela indique aussi que la succession de Charles n'était pas encore complètement réglée à cette époque. Ayant reçu le produit de la vente de sa terre, La Vérendrye se rend à Québec et se prépare à son voyage.

Mais voilà que le 9 novembre, devant François Genaple, notaire à Québec, Pierre La Vérendrye s'engage par un contrat de mariage avec Marie-Anne Dandonneau, fille de Louis Dandonneau du Sablé, seigneur en partie de l'Ile-Dupas et du Chicot, et de Jeanne-Marguerite Lenoir. 
Les deux jeunes gens avaient dû se connaître à Montréal, entre 1704 et 1707. Louis Dandonneau, habitant de Champlain, puis de l'Ile-Dupas, dont il devint seigneur en 1690, s'était établi à Montréal vers 1704 ou peut-être 1703 , et devait y mourir en 1709.

La Vérendrye, dont la famille habitait la maison seigneuriale de Varennes depuis la mort de son père en 1689, avait d'abord fait un séjour à Montréal de 1692 à 1695, avec sa mère et ses frères et sœurs, puis était entré comme cadet dans l'armée en 1697 , et il dut résider dans cette ville d'une façon presque régulière depuis cette époque, en attendant de prendre part à diverses opérations militaires contre les Anglais. Il vécut donc pendant plusieurs années dans le voisinage des Dandonneau.

Dans le contrat de mariage les parents de la future déclarent qu'ils donneront à leur fille, au jour des épousailles, la somme de deux mille livres, et qu'elle possède depuis 1705 une terre près de l'Ile-Dupas, consistant en la moitié de l'Ile-aux-Vaches, plus quatre arpents de front sur une lieue et demie de profondeur dans le fief du Chicot, plus une autre terre de quatre arpents de largeur sur toute la profondeur de l'Ile-Dupas.

Ils lui donnent de plus deux autres lopins de terre dans la même île, l'un de trois arpents de largeur, l'autre de deux. La terre du Chicot n'a pas de culture, mais celles de l'Ile-Dupas ont de seize à dix-huit arpents de terre labourable.

Quant au futur, il donnera à son épouse, au jour du mariage, la somme de deux mille livres de douaire.

Ce contrat de mariage, à la veille d'une longue absence, n'a rien en soi qui doive nous surprendre; il était destiné à resserrer les liens qui unissaient les deux jeunes gens et à adoucir les amertumes de la séparation. Mais le texte de ce document et celui de la vente du 27 octobre nous obligent à faire des constatations plutôt extraordinaires.

$\mathrm{Au} 27$ octobre, on relève des formules comme celles-ci : « désirant passer en l'ancienne France pour s'y établir plus avantageusement qu'en ce pays....», et: «la faculté de reméré sera nulle, s'il repasse en ce pays, un an seulement après qu'il sera de retour....».

Sans renoncer à l'idée de revenir parmi les siens, La Vérendrye avait donc considéré comme une possibilité son établissement définitif en France, peut-être en y faisant venir sa future au moment voulu. D'un autre côté, le contrat de mariage n'avait pas été prévu pour une date si rapprochée et il fut décidé dans l'espace de quelques jours. 
Comme, en effet, Pierre La Vérendrye, encore mineur, a besoin de l'autorisation de sa mère et tutrice, (Jacques-) «René Gaultier de Varennes, son frère aîné, se fait fort du consentement que la dame de Varennes, leur mère, donne au présent contrat de mariage sur les conventions stipulées ci-après, suivant la déciaration qu'elle lui en a faite de vive voix à diverses fois; lequel consentement il assure qu'elle ratifiera et qu'elle enverrait si le temps du départ des vaisseaux n'était si prompt et qu'on eût le temps de lui écrire...»», etc.

On peut conclure de ce qui précède:

Premièrement que les relations entre les futurs époux avaient dû être jusque là plutôt discrètes et que la question du mariage n'avait pas été discutée en vue d'une décision rapide;

deuxièmement, que le mariage avait été tout de même envisagé et les termes du contrat discutés, et que Marie Boucher s'y montrait favorable, puisque son fils aîné l'affirme positivement et se fait fort d'obtenir son consentement; enfin, qu'une intervention se produisit, qui brusqua le cours des événements.

Or, cette intervention ne peut être, selon nous, que celle des époux Dandonneau, qui ont trouvé ce moyen sûr de faire revenir au pays le jeune noble devenu soldat, dont le lustre rejaillirait un jour sur leur fille et sur eux. Cela expliquerait en même temps leur extrême générosité vis-à-vis de leur fille aînée. S'il en fut ainsi, il faut convenir qu'ils avaient calculé juste.

On remarquera encore, au sujet de la vente du 27 octobre, que la terre, vendue à Christophe Dufrost de La Jemmeraye, beau-frère de La Vérendrye, restait dans la famille.

Le vendeur se réservait la faculté de reméré, c'est-à-dire le droit de racheter la terre au bout de cinq ans, au même prix qu'il l'avait vendue, soit huit cents livres. Cela semble indiquer que c'était là le terme de son engagement. Disons tout de suite qu'il n'usa pas de son droit, puisque la partie concernée de la Grande Ile de Varennes resta aux mains de sa sœur et que, veuve de Christophe Dufrost, puis de Timothée Sullivan, elle agira plus tard comme unique propriétaire.

Dernière remarque enfin:

La Vérendrye, qui a été nommé enseigne en second en 1706, est appelé dans son contrat de mariage: "Pierre Gaultier, écuyer, sieur de la Veranderye, officier, (c'est-à-dire enseigne) dans le détachement de la Marine en ce pays ».

Toute cette formule a été ajoutée dans la marge et initialée par le notaire et les principaux personnages présents, parmi 
lesquels le gouverneur. Cela semble indiquer que La Vérendrye avait été fait enseigne en pied, et peut-être séance tenante, par le gouverneur général. Nous n'avons pas de date dans les listes pour cette promotion.

Comme soldat, La Vérendrye avait encore besoin de l'autorisation du gouverneur pour passer son contrat de mariage. Celui-ci mentionne donc le « consentement et agrément de Monsieur Philippe de Rigault, marquis de Vaudreuil, lieutenant général pour le Roy en ce pays ». suivant:

Le 12 novembre 1707, le gouverneur lui donne le certificat

Pièce No 15.

(Certificat du gouverneur de Vaudreuil à La Vérendrye. - AN., Colonies, série E, carton 263, dossier La Vérendrye).

Philippe de Rigaud, marquis de Vaudreuil, chevalier de l'ordre royal et militaire de Saint Louis, gouverneur et lieutenant général pour le Roy en la Nouvelle-France;

Nous certifions à tous qu'il appartiendra que le sieur Delaverendrye, enseigne dans les troupes entretenues par Sa Majesté en ce pays, a toujours servi avec distinction dans tous les endroits où il s'est rencontré pour le service du Roy.

En foi de quoi ... - Fait à Québec le 12 novembre 1707.

(Signé:) Vaudreuil.

La Vérendrye dut quitter le Canada dans les premiers jours qui suivirent.

Pour nous éclairer sur les années de France, comme du reste sur toute la carrière militaire de La Vérendrye, il existe aux Archives nationales de Paris un précieux autographe de celui-ci, dont voici le texte:

Pièce No $^{0} 16$.

(Etat des services de La Vérendrye. - 7 octobre 1749. - AN., Colonies, série E, carton 263, dossier La Vérendrye).

Etat en abrégé des services du sieur Laverendrye.

1697. Fait cadet.

1704. Première campagne à la Nouvelle Angleterre.

1705. Autre campagne en Terre Neuve.

1706. Fait enseigne en second.

1708. Fait sous-lieutenant de grenadiers au premier bataillon de Bretagne, en Flandre.

1709. Resté sur le champ de bataille pour mort avec neuf blessures à la bataille de Malplaquet. tagne.

1710. Fait lieutenant d'une compagnie du premier bataillon de Bre-

1712. Fait enseigne en pied pour le Canada.

1729. Fait lieutenant.

1732. Employé aux découvertes du Ouest avec de ses enfants, après l'établissement de six forts et trois découvertes très éloignées dans les prairies, tant par lui que pour (par) ses enfants. 
1744. Relevé de ces postes et découvertes par le sieur Denoyel.

1745. Fait capitaine.

1746. Rétabli dans ces postes du Ouest avec ses enfants.

1749. Fait chevalier de Saint Louis.

1750. Au printemps doit partir de Montréal pour poursuivre les découvertes du Ouest.

A Québéc, ce 7 octobre 1749

Laverendrye.

Nous avons là, pour les années $1708,1709,1710$ et 1712 , d'importants jalons sur cette période.

Voici maintenant une série de courtes pièces inédites qui nous renseigneront davantage sur les activités de La Vérendrye en France:

Pièce $\mathrm{N}^{\circ} 17$.

(Archives Historiques du Ministère de la Guerre, Château de Vincennes, près Paris. - Registre Y B 806, fol. 74).

3 septembre 1709. Compagnie de grenadiers Sieur de Varenne. Veranderie, sous-lieutenant (à la place de) Francheron, sous-lieutenant de grenadiers.

Pièce No 18.

(Lettre du Maréchal de Boufflers au Ministre, ... septembre 1709. Ibid. A 1, 2152).

Je vous envoie ce paquet par le sieur de Cornis, major de Bretagne, prisonnier de guerre, qui s'en va à Paris pour ses affaires particulières. Je ne puis assez vous dire tous les biens qui me reviennent de lui, et avec quelle valeur et quel entendement il s'est comporté dans l'action du 11 (Malplaquet), dans laquelle tout le régiment de Bretagne et $M$. de Berthelot, colonel, ont fait des merveilles ...

Pièce No 19. 1710).

(Demande d'une lieutenance pour La Vérendrye. - AN D2 D1, 8,

Canada, 1710. - Enseignes en pied qui demandent des lieutenances. Le chevalier de Veranderie. Il dit aussi qu'il est enseigne depuis huit ans et n'est point sur les listes.

Pièce $\mathrm{N}^{\circ} 20$.

(Prestation de serment par La Vérendrye en qualité de lieutenant. 6 mars 1710. - AN - Colonies, série E, carton 263. Dossier La Vérendrye).

Nous, Conseiller du Roy, commissaire provincial des guerres au département de Maubeuge, Le Quesnoy, Avesnes, Landrecies en Hainault et dépendances;

Certifions que, conformément à l'édit de mars 1704 et déclarations rendues en conséquence, le sieur Delaverendrye a prêté en nos mains le serment ordinaire de fidélité à cause de la charge de lieutenant au régiment de Bretagne-Infanterie, où il a plu à Sa Majesté de le pourvoir.

Fait le 5 mars 1710. (Signé:) De Mollien.

Pièce No 21. fol. 75).

(Archives Historiques du Ministère de la Guerre. - Registre Y B 807, 
24 janvier 1711. - Compagnie de La Fresnaye. - La Veranderie, lieutenant, (à la place de) Royer, lieutenant de grenadiers.

\section{Pièce $\mathrm{N}^{\circ} 22$.}

(Ordre à La Vérendrye, 23 septembre 1711. - AN - Colonies, série E, carton 263).

Louis-Christian de Montmorency-Luxembourg, chevalier de l'ordre de Saint-Jean de Jérusalem, lieutenant-général du gouvernement des Flandres et des armées de Sa Majesté, gouverneur des ville et citadelle de Valenciennes.

Il est ordonné au sieur Delaverendrye, lieutenant d'aller en guerre sur les ennemis des Deux-Couronnes avec 25 hommes pendant 25 jours.

Fait à Valenciennes le 23 septembre 1711.

(Signé:) Le chevalier de Luxembourg.

Pièce No 23.

(Ordre à La Vérendrye. - Ibid. - 14 octobre 1711).

à Ypres.

Louis de Grimaldy, maréchal de camp ès armées du Roy, commandant

Il est ordonné au sieur Delaverendrye, lieutenant, d'aller à la guerre sur les ennemis des Deux-Couronnes avec 30 hommes. Le présent passeport valable pendant 15 jours.

Fait à Ypres ce 14 octobre 1711. - (Signé:) le B. de Grimaldy.

Pièce No 24.

(Archives du Ministère de la Guerre. - Registre Y B 808, fol. 14).

22 décembre 1711. - Compagnie de La Fresnaye. Bayeul, lieutenant, par abt (abandonnement) de la Verendrie.

Pièce No 25.

(Demande d'enseigne pour La Vérendrye. - AN - Colonies. - D2 D1. - Pièce No 9, fol. 11).

1711. Conseil (de Marine). - Canada. - Demandes.

Enseignes ...

Pour le sieur de Varenne de Laverrandry qui a fait une recrue de 30 hommes pour le Canada et qui était lieutenant dans les troupes de France.

(En marge, on lit:) Recommandé par Madame de Vaudreuil. — Bon.

Pièce No 26.

(Demande d'une enseigne au Canada pour La Vérendrye. - AN Correspondance Générale. - C $11 \mathrm{~A}$, vol. 33, fol. 257-258).

15 février 1712. Le chevalier de Verandrye, lieutenant dans le régiment de Bretagne, supplie de lui accorder une enseigne dans les troupes de Sa Majesté entretenues en Canada, aux offres de faire une recrue pour ce pays.

Il est natif de Canada. Son père y est mort gouverneur des TroisRivières. Son frère a été tué au service de $\mathrm{Sa}$ Majesté en France, étant dans le régiment d'infanterie de Bretagne, et son oncle est mort gouverneur d'Arlon, dans le Luxembourg.

Il a servi six ans, savoir en Canada trois campagnes dans les partis envoyés contre les Anglais, - et trois autres en qualité de lieutenant dans ledit régiment de Bretagne. 
Il a reçu à la bataille de Malplaqué quatre coups de sabre et un coup de fusil au travers du corps et a été prisonnier de guerre pendant quinze mois; ce qui l'a mis hors d'état de pouvoir subsister dans les troupes de terre en France, à cause des grandes pertes qu'il a faites, et ne recevant aucun secours de son pays.

Il rapporte des copies des certificats de ses services du Maréchal de Luxembourg, lieutenant-général, de $M$. de Grimaldy, maréchal de camp, de M. le Marquis de Vaudreuil de Canada, et M. Berthelot, colonel du régiment d'infanterie de Bretagne.

(Endos: Canada. Conseil. (non signé). Pour Mr de la Verendrye, 30 hommes de recrue).

Pièce N $^{\circ} 27$.

(Brevet d'enseigne de La Vérendrye. - AN - Colonies - D2 C, vol 57 , fol. 57 ).

Brevet d'enseigne pour le sieur de Varennes de la Veranderie, du 24 mai 1712.

De par ke Roy. Sa Majesté ayant fait choix du sieur de Varennes de La Veranderie pour servir en qualité d'enseigne dans une des compagnies d'Infanterie du détachement de la Marine qu'elle entretient dans la Nouvelle France, mande Sa Majesté au sieur Marquis de Vaudreuil, gouverneur et son lieutenant-général audit pays, de le faire recevoir et reconnaître en ladite qualité de tous ceux et ainsi qu'il appartiendra.

Fait à Versailles le vingt quatrième mai mil sept cent douze.

Signé: Louis, et plus bas Phélypeaux.

et au dos est écrit: Enregistré au contrôle de la Marine et des Fortifications de la Nouvelle France, à Québec, le vingt septième octobre mil sept cent treize.

(Signé) De Monseignat.

Pièce No 28.

(Lettre du Ministre à La Vérendrye. - AN - Colonies, série B: Ordres et Dépêches du Roi, vol. $34-2-1712$, fol. 363 . - Classé sous le titre: « Lettres de Plaisance »).

Le sieur Varenne de la Veranderie.

A Marly, le 21 juin 1712. - Comme le Roy a bien voulu vous accorder une enseigne dans les troupes qu'elle entretient en Canada, il est nécessaire que vous passiez cette année en cette colonie. Le compte que je lui ai rendu de vos services a été cause qu'il vous a fait cette grâce, à laquelle je suis bien aise d'avoir contribué, persuadé que (vous) servirez bien et que je ne recevrai point de plainte de votre conduite.

Tels sont les principaux documents que nous avons sur la vie militaire de La Vérendrye en France.

Nous y ajouterons quelques commentaires et corrigerons quelques erreurs que nous trouvons dans les livres et les revues.

Quand La Vérendrye arriva en France, la guerre sévissait avec violence, particulièrement dans les Flandres, et le pays était aux abois, attaqué à la fois par l'Angleterre, l'Espagne et la Hollande. On manquait de soldats, de munitions, d'argent, de tout. Il ne semble pas que La Vérendrye ait été diminué d'un 
grade, comme c'était la pratique courante quand un soldat passait des colonies dans la métropole et vice versa, comme ç'avait été le cas pour son frère Louis. A cause de la guerre, la tendance était plutôt de donner des promotions aux bons soldats.

S'il faut en croire l'état de service de 1749 , cité précédemment, La Vérendrye fut promu dès la première année, car il ne put guère arriver en France avant la fin de janvier 1708. La pièce $\mathrm{N}^{\circ} 17$ semble indiquer cependant, comme date de cette promotion, le 3 septembre 1709, ce qui est probablement plus exact. En 1749, quarante ans après les faits, il était facile à La Vérendrye de se tromper de quelques mois. De toute façon, il est nommé sous-lieutenant dans ce régiment de Bretagne où son oncle Charles avait été lieutenant-colonel pendant vingt-cinq ans, où son frère était tombé et où sans doute il avait demandé à être incorporé.

Le 11 septembre 1709 avait lieu la fameuse bataille de Malplaquet, qui fut une mêlée comme on en connaît peu dans l'histoire.

De grands chefs militaires se rencontraient ici: le marquis de Villars pour la France, et, contre elle, le duc de Marlborough pour l'Angleterre et le prince Eugène de Savoie-Carignan pour les Impériaux. On fit des prodiges de valeur de part et d'autre, et 25,000 hommes restèrent sur le champ de bataille.

Le régiment de Bretagne, qui s'était déjà signalé depuis 1701 dans la guerre d'Italie, et qui était commandé à Malplaquet par M. de Berthelot, colonel, «fit des merveilles », comme le marquis de Boufflers l'écrivait au Ministre quelques jours après.

La Vérendrye reçut huit coups de sabre et une balle lui traversa le corps: neuf blessures en tout, dont cinq étaient graves. Il fut « laissé pour mort » et guérit contre toute espérance.

Tous ces renseignements sont tirés de documents officiels, des états de services de La Vérendrye, et de brèves allusions dans les lettres de celui-ci, de ses fils et de quelques autres personnages.

La Vérendrye ajoute, au 15 février 1712 (Pièce $\mathrm{N}^{\circ} 26$ ), après avoir mentionné ses blessures à Malplaquet, qu'il fut quinze mois prisonnier de guerre. Des auteurs ont écrit qu'il fut fait lieutenant sur le champ de bataille. La première de ces affirmations demande une explication, la deuxième une rectification.

Il est certainement impossible qu'il ait été prisonnier quinze mois de suite après Malplaquet, puisque, le 6 mars 1710, il prêtait serment comme nouveau lieutenant (Pièce $\mathrm{N}^{\circ} 20$ ), et que, avant cette date, il avait fait sa demande de promotion (Pièce $\mathrm{N}^{\circ} 19$ ). 
Après Malplaquet, il n'a donc pu être prisonnier plus de cinq mois. Il n'y a pas de place, d'un autre côté, pour plus de neuf mois, entre le 6 mars 1710 et le 24 janvier 1711 (Pièce $\mathrm{N}^{\circ} 21$ ), où on le trouve à son poste de lieutenant. Il faut conclure, ou que La Vérendrye fut prisonnier quinze mois au commencement de son séjour en France, 1708-1709, ou qu'il fit deux ou trois stages en cette qualité avant et après Malplaquet.

Une autre remarque s'impose ici. La Vérendrye, à l'occasion de sa demande de 1710, est dit « enseigne en pied », alors que nous savons qu'il était sous-lieutenant. C'est que le grade de souslieutenant n'exista que par intermittences, particulièrement en temps de guerre, et qu'il fut porté par des enseignes en pied, qui gardèrent ce dernier titre seulement à la fin de la campagne, passant ensuite directement au grade de lieutenant.

C'est ce qui arrivait régulièrement au Canada, où le grade de sous-lieutenant était pratiquement inconnu.

Quant à avoir été fait lieutenant sur le champ de bataille de Malplaquet, la chose est certainement inexacte, comme le prouvent les pièces 19 et 20 .

Notons en passant que l'indication «Canada, 1710 », sur la pièce $\mathrm{N}^{\circ} 19$, signifie que La Vérendrye appartenait toujours à l'armée canadienne et était considéré comme tel.

Une autre constatation intéressante, c'est que, dans la pièce $\mathrm{N}^{\circ} 17$, on voit La Vérendrye nommé sous-lieutenant le 3 septembre 1709, juste avant Malplaquet, «dans la compagnie du sieur de Varenne ».

Certains auteurs ont cru voir dans ce capitaine un cousin de La Vérendrye ou même son frère Louis. Il n'en est rien. Ce M. de Varenne était natif de Saint-Chamond, dans le Lyonnais, comme le prouvent plusieurs documents, dont nous extrayons ceci :

Pièce No 29.

(Archives Historiques du Ministère de la Guerre, Vincennes, X B 59, régiment de Bretagne).

Second bataillon du régiment de Bretagne. - Etat du 15 octobre 1705 . - M. de Varenne, capitaine, commandant ledit bataillon.

Natif d'auprès de Lyon, dans le Lyonnais, âgé de trente huit ans. A commencé à servir dans la compagnie des cadets, 1688; fait capitaine au régiment de Poitou, août 1690 ; placé dans le second bataillon du régiment de Bretagne à sa création, le 1er mars $1701 \ldots$

Pièce No 30.

(Ibid.).

Etat des officiers qui composent l'ancien bataillon du régiment de Bretagne, 1709. Deuxième bataillon: $M$. de Varenne, capitaine de grena- 
diers de la première compagnie. Natif de Saint-Chamond en Engenois ${ }^{34}$. (A servi tant) en qualité de cadet que de compagnies détachées... Commission du 12 août $1690 \ldots$...

Pièce No 31.

(Ibid.).

Le chevalier de Varenne, (apparemment fils du précédent), souslieutenant de la compagnie, natif de Saint-Chamond, âgé de 16 ans. Souslieutenant en 1708.

Des renseignements reçus d'autres sources nous permettent, croyons-nous, de rattacher ces personnages aux Burignot de Varennes, qui habitent aujourd'hui les départements de l'Ain et de la Charente ${ }^{35}$.

La seule difficulté est que, dans les pièces qui précèdent, La Vérendrye mentionne le premier bataillon, tandis que les textes concernant le sieur de Varennes parlent du deuxième bataillon. Mais ces mêmes textes mentionnent la première compagnie, ce qui semble contradictoire. L'explication est probablement que la première compagnie, que commandait le sieur de Varennes, a pu appartenir à des moments différents à deux bataillons du même régiment ou qu'il y a eu confusion quelque part.

Les pièces 22 et 23 nous montrent La Vérendrye en pleines opérations militaires jusqu'à la fin d'octobre 1711.

Puis, vers le 22 décembre, il quitte définitivement le régiment de Bretagne (c'est le sens de l'ancien mot «abandonnement »), pour se préparer à retourner dans son pays.

Mais dans l'intervalle entre sa décision et son retour parmi les siens, nous verrons se dérouler des événements du plus haut intérêt, auxquels se rapportent les pièces $\mathrm{N}^{\circ} 25$ et les suivantes.

A l'aide de ces documents et avec quelques notions de l'histoire de cette période, on devrait pouvoir reconstituer ainsi la suite des événements:

La bataille de Malplaquet, bien que favorable aux alliés européens, leur avait été terriblement désastreuse, et elle avait fait voir en même temps une France malheureuse plus unie que jamais à son roi.

D'autre part, la diplomatie de Louis XIV et d'autres facteurs qu'il serait trop long d'énumérer ici avaient réussi à desserrer les liens qui unissaient l'Angleterre à ses alliés.

En conséquence, les pourparlers secrets entamés entre la France et l'Angleterre en vue d'une paix séparée se poursui-

34 《 Engenois 》 est certainement une erreur. Saint-Chamond est dans le Lyonnais, comme le dit la pièce précédente. 192-193.

35 Voir notre article dans le BRH (octobre-novembre-décembre 1956), 
vaient avec des chances sérieuses de succès, tandis que sur le front des Flandres, la guerre se déroulait avec un mouvement ralenti et que le besoin des soldats se faisait de moins en moins sentir.

Durant la deuxième moitié de 1711, La Vérendrye, qui n'était plus employé qu'à des escarmouches et à des opérations de détail, et qui se rendait compte qu'il avait plus à perdre qu'à gagner à rester en France, se détermina à retourner au Canada au printemps de l'année suivante. Il fit sa demande, qui lui fut accordée sans difficulté. Le 22 décembre, on voit qu'un lieutenant Bayeul l'a remplacé dans la compagnie de La Fresnaye (Pièce $\mathrm{N}^{\circ} 24$ ).

Mais les grades acquis pendant la guerre disparaissaient automatiquement une fois la campagne terminée; et de plus, le grade d'enseigne, qui lui avait été donné par le gouverneur avant son départ du Canada, n'avait pas été reconnu ni confirmé par le roi. L'héroïque soldat de Malplaquet redevenait, de lieutenant, simple cadet. Et il se retrouvait plus pauvre en deniers et en grades militaires qu'il n'était avant le départ.

Cependant, fort de la protection de Madame de Vaudreuil, qui était à Paris depuis l'automne de 1709 , au poste de confiance de sous-gouvernante des enfants royaux, il demanda une commission d'enseigne pour le Canada octroyée par la Cour (Pièces 25 et 26 ).

On la lui promit à une condition.

A cette époque, si la France ne se sentait plus aussi menacée, il n'en était pas de même du Canada, dont dépendaient l'Acadie et Terreneuve, et où le besoin de soldats était grand, les opérations se continuant de ce côté avec plus de férocité que jamais.

Il fut entendu que La Vérendrye aurait sa commission d'enseigne, mais qu'il devait recruter trente soldats pour le Canada. C'était une besogne facile. Il était encore lieutenant dans l'armée de France. Sa bravoure connue et son caractère enthousiaste avaient dû aussi le rendre populaire. Il n'eut qu'à s'adresser aux soldats avec lesquels il était ou avait été en contact. Il eut bientôt recueilli ses trente recrues et, la recommandation de Madame de Vaudreuil ayant opéré de son côté, le roi, sur la demande d'enseigne, écrivait: «Bon ». Cela se passait avant la fin de 1711 (Pièce $\mathrm{N}^{\circ} 25$ ).

Il fallait remplir une demande officielle accompagnée des Etats de services du pétitionnaire et destinée à son dossier militaire. La Vérendrye remplit cette formalité le 12 février 1712. C'est la pièce $\mathrm{N}^{\circ} 26$ : «le chevalier de Verandrye, lieutenant dans le régiment de Bretagne, supplie... de lui accorder une enseigne dans les troupes que Sa Majesté entretient au Canada, aux offres de faire une recrue pour ce pays...». 
Le 27 avril, le Ministre écrivait à Madame de Vaudreuil: "J'ai reçu votre lettre et les deux placets 》, ce qui semble bien se rapporter à la recommandation ci-dessus. ${ }^{36}$

Le 24 mai, son brevet était signé par le roi. (Pièce $\mathrm{N}^{\circ} 27$ ).

Mais il est un détail intéressant qu'aucun historien ne semble avoir encore remarqué. C'est que, au lieu de se diriger immédiatement vers le Canada, il avait ordre de se rendre à Terreneuve, pour y conduire les soldats qu'il avait recrutés.

Voici d'autres documents qui complètent, à ce sujet, le $\mathrm{N}^{\circ} 28$.

Pièce No 31 .

(Archives Nationales. Lettres de Plaisance. Ordres et dépêches du Roi, série B, vol. 34-1, fol. ... - 1712).

(Le Ministre à) M. de Beauharnois, (qui se trouvait alors en France). A Versailles, 4 mai 1712.

Je suis bien aise que le sieur de Varennes ait mené à Rochefort une recrue de vingt soldats pour le Canada.

Vous avez bien fait de les faire passer en Olleron jusqu'à leur embarquement.

\section{Pièce No $31 b$.}

(Ibid., fol. 26-27).

(Le même à) M. de Beauharnois. A Marly, le 18 juin 1712 .

J'ai ordonné au trésorier de remettre à son commis à Rochefort pour 63333 livres d'assignations, suivant l'ordre de fonds qui est ci-joint, tant pour servir à tous les achats qu'au paiement de l'habillement des seize soldats de recrues qui ont été embarqués pour le Canada sur les navires «La Louise», de Rochefort, et «L'Heureux Retour», de La Rochelle, et pour celui des trente autres de recrues qui doivent passer sur « Le Héros 》. Comme Sa Majesté souhaite que les deux compagnies de l'Acadie qui sont dans l'île Doléron se rendent au lieu de leur destination, je vous prie de prendre vos mesures pour qu'elles passent sur le vaisseau du Roi « Le Héros 》 avec tous les officiers, afin qu'elles se rendent à Plaisance.

Je vous prie de penser aussi au passage des officiers du Canada qui sont en France et qui doivent se rendre incessamment à La Rochelle.

Je vous en enverrai la liste au plus tôt.

Il y a encore la dame de Noré et la veuve Dusablé et sa mère qui s'en retournent au Canada. Vous me ferez plaisir de faire votre possible pour les y faire passer.

Je vous prie de presser le plus que vous pourrez le départ de ce vaisseau, car je vous enverrai au premier jour tous mes papiers.

\section{Pièce No 31c.}

(Ibid., fol. 53, verso).

(Le même à) M. D'Alogny. A Marly, le 25 juin 1712 .

Il a passé cette année seize hommes de recrue pour le Canada sur le vaisseau "La Louise». Il y en passe encore trente dans le vaisseau du Roi «Le Héros». Il serait à souhaiter qu'elles fussent en plus grand nombre, mais les temps durs et difficiles en sont la cause. Ils empêchent aussi Sa Majesté de se déterminer à remettre les compagnies de ses troupes qui sont dans la Colonie, sur le pied de cinquante hommes.

${ }^{36}$ AN. Série B. Ordres et dépêches du Roi, vol. 34, fol. 2.1712. 
Pièce No 31.

(Fbïd., p. 11:5, ,

(Le même à) M. de Costebelle. Marly, le 27 juin 171

Elle '(Sa Mijesté) a donné ordre de faire passe: Plaisance le le compagnies de la Ronde Denis et de Saint-Vincent, de la garnison de l'Acadie, avec les soldats de recrue qui sont à l'île d'Oléron.

Ces troupes dörvent être embarquées sur le vaisseau « "Héros».

Ce wera un mecours et une augmentation qui vous mettra é état de ne point craindre les entreprises des ennemis.

Pièce No 3 lie.

(Ibid., fol. 95).

(Le mềme ầ) M. Ie Marquis de Vaudreuil. A Marly, le 5 juillettioni2.

Mionsieur,

J'écris à $M$. de Beauharnois de faire passer sur le vaisseau du' Roi "Le Héros» les deux compagnies de l'Acadie, qui sont destinées poưr lá. colonie de Plaisance, quand même ce vaisseau n'irait point; et en câs', Sa Majesté souhaite que vous gardiez en Canada ces deux compagnles jusqu'à nouvel ordre.

Je suis ......, iete.

Pièce No $31 f$.

(Ibid., fal. ......).

(Le même à) M. de Beauharnois. A Marly, le 5 juillet 1712 .

....Q Quand même « Le Héros 》n'irait point à Plaisance, vous devez faire passer sur ce vaisseau les compagnies qui sont destinées pour ce lieu, afin qu"elles se rendent au moins en Canada.

La Vérendrye, d'après ces documents, a donc ordre de se diriger vers Plaisance. Tout semble indiquer que les choses se passèrent comme prëvu et que La Vérendrye et les trente recrues qu'il accompagnait arrivèrent sans encombre.

Que ces recrues aient été destinées à Terreneuve plutôt qu'au Canada et que le jeune officier ait été chargé de les conduire à destination, rien de plus naturel, car, d'un côté, si le Canada avait eu de graves alertes en 1710 et 1711 , avec Nicholson vers le lac Champlain et la flotte de Walker sur l'Atlantique, il n'était pas menacé en 1712, tandis que dans les colonies de l'Acadie et de Terreneuve on se battait ferme depuis plusieurs années et le besoin de soldats avait plutôt augmenté que diminué ${ }^{37}$.

D'un autre côtê, il fallait un officier pour les accompagner et les commander en route, et qui pouvait mieux le faire que le jeune enseigne qui avait gagné leur confiance et avait été assez persuasif pour les décider à quitter leur patrie et à aller se battre, non plus seulement sur ses frontières, mais par delà l'océan ?

37 En 1711, selon Rameau de Saint-Père, La France aux Colonies, p. 87 , il n'y avait à Plaisance et sur la côte de Terreneuve, outre les pêcheurs hivernants, que 66 habitants en état de porter les armes. La plupart des soldats devaient venir de Franee ou du Canada. 
Parti de Rochefort après le 5 juillet, sur le vaisseau «Le Héros », La Vérendrye dut arriver à Terreneuve dans la première quinzaine d'août. En mettant les pieds sur le sol d'Amérique, l'officier se retrouvait en pays connu, puisqu'il s'était battu dans ce même territoire en $\mathbf{1 7 0 5}$.

Il n'y fit qu'un très bref séjour. Il est certain qu'il était pressé de se retrouver parmi les siens et auprès de sa fiancée après une si longue absence et après avoir vu la mort de si près, à Malplaquet et peut-être ailleurs.

Le roi lui ordonnait de passer au Canada dans le courant de l'année: il n'eut pas à se faire prier, et, sa mission accomplie au sujet des recrues, il prit le premier bateau en partance pour Québec. Il dut y arriver au plus tôt dans le courant de septembre, peut-être en octobre.

Faisons ici quelques remarques, qui mettront au point certains détails.

Pourquoi La Vérendrye revient-il en son pays natal en cette année 1712 ?

Il faut répondre que plusieurs circonstances ont contribué à ce retour à ce moment. Tout d'abord, le texte de la vente de sa terre de Varennes semble indiquer qu'il s'était engagé pour cinq ans. Or ce temps expirait en 1712 et il arrivait juste à temps pour profiter des pleins droits qu'il s'était réservés: un an après sa majorité.

Ensuite, la guerre achevait en Europe et le traité d'Utrecht se préparait dans le secret, ce qui permettait à la France de se libérer du fardeau de l'entretien d'une armée qui épuisait ses finances.

Enfin, La Vérendrye n'avait pas trouvé en France ce qu'il y cherchait, bien au contraire.

Il était parti avec l'espoir de «trouver de l'emploi et de s'avancer, ce qu'il ne pourrait faire en ce pays ». Il revenait au Canada parce que son grade de lieutenant en France l'obligeait à un certain décorum et que ses appointements ne lui permettaient pas de satisfaire à ces exigences. Il aurait fallu prendre sur ses fonds personnels : or, il n'en avait pas.

Pour ce qui est des grades militaires, avait-il gagné davantage ? Nommé enseigne en 1707 par le gouverneur, il revenait avec une commission du roi pour le même grade. Cette nomination par la Cour était un léger progrès, mais il y serait probablement parvenu également s'il était resté au Canada.

Des auteurs ont jeté le blâme sur la mère-patrie, la France, et ont même prononcé le mot d'ingratitude. Ne soyons pas si 
sévères. Comme d'autres historiens l'ont écrit plus justement, la France était saignée à blanc: son trésor était vide. Chacun payait de sa personne, sans se rendre compte de l'immensité de son sacrifice. C'était le patriotisme chevaleresque de cette époque.

Si la France eût voulu faire davantage pour ses héros, elle ne l'eût pas pu. Il faut ajouter que tout cela était conforme aux conceptions et aux habitudes de cette époque et que personne ne songeait à s'en plaindre.

Nous remarquerons encore que La Vérendrye, dans les pièces 19 et 26,1710 et 15 février 1712 , reçoit le titre de «chevalier 》, que porte quelquefois son père et qu'il donnera plus tard à son fils Louis-Joseph, selon une habitude assez peu comprise qu'avaient les familles nobles de donner le titre de chevalier à un de leurs fils. Ce sont les deux seules occasions où nous le rencontrons avec ce titre.

Venons-en à la suite des événements et à la conclusion de cette étude sur La Vérendrye en France.

Après son séjour à Terreneuve, nous retrouvons celui-ci pour la première fois au Canada le 24 octobre 1712 , alors qu'il ratifie le contrat de mariage qu'il avait fait le 9 novembre 1707, avant son départ pour la France, avec Marie-Anne Dandonneau du Sablé, fille du seigneur de l'Ile-Dupas. Cetière.

Cette ratification fut faite à Québec, devant le notaire La

Il devait être de retour depuis peu de jours.

Le lendemain, il obtenait du gouverneur Vaudreuil la permission de contracter mariage et, quatre jours plus tard, le 29 octobre, la cérémonie avait lieu dans l'église de Québec.

La Vérendrye était redevenu canadien.

Telle est la suite des événements connus sur le séjour de La Vérendrye en Europe.

Nous croyons qu'il n'est pas sans intérêt de signaler, entre beaucoup d'autres, quelques mentions des blessures de La Vérendrye et de leurs cicatrices. Elles constituent un éloquent témoignage de son extraordinaire bravoure.

- 15 février 1712 (Pièce $\mathrm{N}^{\circ} 24$ ) : «... a reçu à la bataille de Malplaquet quatre coups de sabre et un coup de fusil au travers du corps... ».

- Octobre 1722. Liste des officiers qui servent en Canada: «Enseignes en pied. ... Le sieur de Laveranderie: il a servi en France et a été blessé à la bataille de Malplaquet, et je ne lui vois que de bonnes qualités ». 
- 1er août 1731. La Vérendrye au Ministre: «... cinq grandes blessures sur le corps .... .

- Janvier-février 1734. Mémoire de La Vérendrye, 17331734: «...Je restai (au fort Saint-Pierre) encore sept jours pour me remettre de la fatigue du voyage, ayant ressenti mes anciennes blessures, qui pensèrent m'arrêter en chemin ... ».

-9 mai 1734. Ibid.: «... Je leur fis voir (aux chefs cris et monsonis) les blessures que j'avais reçues dans la bataille de Malplaquet. Ils en restèrent dans l'étonnement... ».

-24 octobre 1744. Beauharnois au Ministre: «... neuf blessures sur le corps ....».

- 7 octobre 1749 (Pièce $\mathrm{N}^{\circ} 16$ ). Etat des services de La Vérendrye: "Resté sur le champ de bataille pour mort avec neuf blessures à la bataille de Malplaquet ».

Les expressions : "laissé pour mort 》, et autres de ce genre, indiquent la gravité des coups reçus. Le nombre des blessures: neuf, dont cinq plus graves que les autres, laisse voir la détermination de mourir plutôt que de céder.

Finissons sur ce sujet en citant des passages d'une lettre d'un missionnaire aux Sioux, qui connut et admira La Vérendrye et recommanda ses plans pour la découverte de la mer de l'Ouest. Elle résume, mieux que tout autre texte que nous connaissons, les faits que nous avons énumérés. Elle nous montre en même temps l'opinion qu'un homme impartial et clairvoyant pouvait déjà se faire de La Vérendrye au commencement de sa carrière de découvertes.

Pièce No 32. 65-67).

(BN - Mss français. - Nouv. Acquisitions. - Margry, 9286, fol.

3 novembre 1728. Lettre du Père de Gonnor, jésuite, missionnaire des Sioux, au Marquis de Beauharnois.

J'ai rencontré (à Michilimakinac) M. de la Vérandrye, revenant du Nord (Kaministiquia et Nipigon), qui m'a confié pour vous les présenter les mémoires pris sur les rapports de plusieurs sauvages ...

Cet officier a l'honneur d'être connu de vous... Son père a été gouverneur des Trois-Rivières...; son frère aîné, capitaine dans le régiment de Bretagne, fut tué en Italie. Il a lui-même servi avec honneur pendant six ans dans ce régiment en qualité de lieutenant. Il s'est trouvé à la bataille de Malplaquet, où il reçut un coup de fusil au travers du corps avec cinq coups de sabre, dont il porte encore les glorieuses cicatrices. Quoique fort jeune, il se trouvait en passe de parvenir à faire son chemin, mais, le bien et la fortune lui manquant, il demanda à venir en son pays continuer son service.

On y consentit, mais on ne put lui accorder pour lors qu'une enseigne, avec promesse de lui rendre son grade à la première occasion. Sur ces entrefaites, ses protecteurs étant morts, il a été oublié, quoiqu'il fût depuis 
vingt ans et plus dans le service, sans qu'on puisse, grâces à Dieu, rien lui reprocher, sinon qu'il ne s'est pas rendu importun par ses demandes.

Il semblerait juste que si la Cour veut faire quelque entreprise sur les mémoires qu'il a fournis, on lui en donnât le commandement, ses services passés, et en France et en Canada, ceux qu'il est en mesure de rendre à l'âge de quarante ans, joints à ses blessures, et le temps depuis lequel il sert $\mathrm{Sa}$ Majesté, lui faisant espérer qu'on pensera à son avancement...

Il ne reste qu'à dire un mot de certaines démarches que fit plus tard La Vérendrye en vue d'un nouveau voyage en France, mais qui furent bientôt oubliées dans une entreprise bien différente.

Le 30 octobre 1724, Vaudreuil et Beauharnois demandent au Ministre un congé ou permission pour les sieurs de la Veranderie, de Raimond, Céloron et La Pérade, qui désirent passer en France pour affaires indispensables ${ }^{38}$.

Sur la lettre de Vaudreuil, le Ministre écrivit: «Bon », puis, le 29 mai 1725, il lui écrivit pour lui envoyer la permission accordée ${ }^{39}$.

Pour une raison ou pour une autre, plusieurs des intéressés, parmi lesquels La Vérendrye, ne purent profiter de leur congé.

Le 28 septembre 1726, Beauharnois et Dupuy revenaient à la charge pour les sieurs La Vérendrye, Céloron et Dugué, puis encore le 20 octobre suivant, en ajoutant le sieur Amariton ${ }^{40}$.

Sans opposer un refus direct, le Ministre répondit cette fois à Beauharnois, le 22 avril 1727, que les intéressés feraient mieux de ne pas profiter du congé qui leur avait été accordé précédemment ${ }^{41}$. La Cour motivait ce conseil en faisant remarquer que si ceux qui désiraient se rendre en France le faisaient avec le désir de travailler à leur avancement, ils prenaient un mauvais chemin pour y arriver.

Etait-ce là l'intention de La Vérendrye et des autres ? Plusieurs l'ont supposé, pour La Vérendrye au moins, et probablement avec raison.

Dix-huit ans s'étaient écoulés depuis Malplaquet, et, malgré les promesses, rien n'avait encore été fait pour récompenser La Vérendrye ou même seulement pour lui rendre les grades qu'il avait conquis. Puisqu'on l'oubliait, n'aurait-il pas plus de succès en allant lui-même plaider sa cause ?

38 AN. Colonies. Corr. gén. C 11, vol. 46, fol. 115.

39 Ibid., série $B$, vol. 48 , fol. 841 .

40 Ibid., C 11, vol. 48, fol. 58; et D 2 D 1, pièce 14 .

41 Ibid., série B, vol. 50 , fol. 490 . 
Quoiqu'il en soit, contrairement à ce qui a été écrit, il ne fit pas le voyage de France. Avant que la lettre du Ministre ne fût arrivée au Canada ou même écrite, il avait pris une autre décision qui allait changer la face de sa vie.

Depuis un an, son frère Jacques-René était commandant à Kaministiquia ou poste du Nord, dont dépendait Nipigon, sur le lac Supérieur, et où il avait succédé à Jean-Baptiste Saint-OursDeschaillons. Il dut faire des propositions à son frère à l'automne de 1726, après le départ des bateaux.

Le 28 mars 1727, devant Jean-Baptiste Adhémar, notaire à Montréal, une double société était organisée pour l'exploitation de ce poste, comprenant d'un côté Jacques-René Gaultier de Varennes, "commandant au Poste du Nord », et Jean-Baptiste Godefroy; et de l'autre, Pierre Gaultier de La Vérendrye, « second commandant » au même poste, et Joseph-Marie Lécuyer.

La vocation $d u$ « Découvreur » naissait.

Trois mois plus tard, La Vérendrye partait pour l'Ouest et la côte nord du lac Supérieur, où il allait faire un noviciat de quatre ans avant de se lancer dans la grande découverte.

VI. Charles-Gabriel Philibert De L'INGranderie, Cousin DE LA VÉRENDRYE, MEURT À QUÉBEC EN 1734, AU RETOUR D'UN VOYAGE EN FRANCE.

Fils de Pierre-Paul Philibert de l'Ingranderie et de Perrine Gaultier, sœur de Charles Gaultier de La Veranderie et de René Gaultier de Varennes, Charles-Gabriel était un cousin germain de La Vérendrye.

Ses parents s'étaient mariés à Bécon le ler février 1663. Voici le texte de ce document:

Pièce No 33.

(Mariage de Pierre-Paul Philibert de l'Ingranderie et de MariePerrine Gaultier. - Des registres de la paroisse de Bécon, Maine-et-Loire). Le 1er février 1663 ont été célébrées dans l'église de Bécon les épousailles de Pierre Philibert, écuyer, sieur de l'Inglanderie, fils de défunt René Filbert, sieur de la Garde, et de damoiselle Catherine Bridonneau, son épouse; - et damoiselle Perrine Gaultier, fille de Pierre Gaultier, sieur de la Verranderie, et de Bertrande Gourdault; - en présence dudit sieur Gaultier, soussigné.

(Signé:) Gautier - Philbert Lingrandri Thomas - De la Barre

Les Philibert étaient-ils nobles ? C'est peu probable, bien qu'on leur donne ici le titre d'écuyers, car en plusieurs occasions, 
comme pour Adam-Pierre et les autres Gaultier, on leur donne tantôt le titre d'écuyers et tantôt celui de nobles hommes.

Charles-Gabriel déclarait dans son testament (Pièce 36) être natif de Saint-Martin-de-la-Place, diocèse d'Angers, et il avait dû naître vers 1673 ou 1674, selon son acte de sépulture et plusieurs autres documents, mais nous n'avons pas trouvé son acte de baptême au registre de cette paroisse, bien que nous ayons ceux de sa sœur et de son frère aîné, baptisés à Bécon en 1664 et 1665.

Nous lui connaissons cinq frères et sœurs.

Charles-Gabriel paraît pour la première fois au Canada le 30 octobre 1706 , alors qu'il est parrain à Varennes, de Joseph Dufrost de La Jemmeraye, son cousin du 2 au 3. On l'y qualifie d'enseigne.

Il n'est pas nommé en 1706, dans une liste du 9 juin, qui donne les noms des officiers passés au Canada cette année-là. Il a donc dû arriver en 1705 ou avant, ou encore en 1706, après la date précitée. On le retrouve à Montréal le 7 août 1712, au mariage de son cousin, Jacques-René Gaultier de Varennes. On le dit officier, c'est-à-dire enseigne.

Nous le voyons encore dans divers documents en 1713, 1714, $1718,1722,1725$, etc., toujours avec le titre d'enseigne, mais cependant il ne tint ce grade que du gouverneur, comme le prouvent les documents suivants :

Pièce No $^{\circ} 34$.

(AN - Colonies. - C $11 \mathrm{~A}, 120$ )

Liste des officiers de guerre qui servent en Canada, dressée par le gouverneur de Vaudreuil, 26 octobre 1722, à Québec:

Enseignes en second:

Le sieur Philbert, 45 ans. Il est fort bon officier en état de servir et qui mène une vie fort réglée.

Pièce No 35.

(AN - Colonies. - Alphabet Laffilard, - D2 C, 222, fol. 629).

Philbert.

Enseigne en second, Canada, 26 mai 1723.

Enseigne en pied, Canada, 23 avril 1726.

Mort.....

Remplacé, 1er avril 1735.

On le trouve ainsi mentionné dans de nombreuses listes et affaires, militaires et autres.

Le 26 septembre 1724, au cours d'une altercation de Timothée Sullivan, son cousin par alliance, avec Antoine Poudret, à 
Montréal, il arrive à séparer les combattants et à « amener ledit sieur Sylvain ... ${ }^{42}$.

Le 19 septembre 1729, à Montréal, il est parrain de CharlesMadeleine d'Youville, fils de sa petite cousine Mme d'Youville. Chose curieuse, on le nomme dans l'acte «Sarle Philibert » et il signe «S. Philibert ». Il devait prononcer ainsi.

Une liste des compagnies servant au Canada en 1729 nous donne:

«Compagnie de Lagauchetière: Capitaine Lagauchetière, lieutenant Verchère, enseigne Philbert, enseigne en second Demuy. quart $\gg{ }^{43}$.

"Québec, 15 octobre 1729. (Signé) : Tilly. Approuvé: Hoc-

Cette année-là, Charles-Gabriel reçut la nouvelle de la mort d'un parent en France et demanda à y passer pour recevoir la succession. La demande fut envoyée à la Cour par Beauharnois le 25 octobre 1729 et celle-ci l'agréa le 17 janvier $1730^{44}$. Il dut y aller à l'automne de cette année-là.

En 1731 et 1732 , permission du Ministre de prolonger son séjour en France ${ }^{45}$. Selon toutes les apparences, il ne revint au Canada qu'en 1734, et ce fut pour y mourir.

Pièce No 36.

(Testament de Charles-Gabriel Philibert, 3 septembre 1734. - Greffe de Barolet, notaire royal. - Archives de la Province de Québec, Hôtel du Gouvernement, Québec).

3 septembre 1734. Fut présent Charles-Gabriel Philibert, officier... natif de Saint-Martin-de-la-Place, diocèse d'Angers, fils de Pierre-Paul Philibert et de Perrine Gautier, vivans ses père et mère, arrivé depuis peu de l'ancienne France sur le vaisseau du Roy le Rubys, duquel il est débarqué indisposé, actuellement logé chez le sieur Joly, maître boulanger ... et malade,... néanmoins sain d'esprit, mémoire et entendement, ainsi qu'il est apparu au notaire et témoins soussignés, lequel ... a fait et dicté son testament en la manière qui suit.

Premièrement, comme vrai chrétien, a recommandé son âme à Dieu, Père, Fils et Saint-Esprit, le priant, quand elle se séparera de son corps, la placer au rang des bienheureux.

Veut et entend le testateur qu'avant toutes choses ses dettes soient payées et torts par lui faits, si aucuns se trouvent, par son exécuteur testamentaire ci-après nommé.

42 Archives du greffe criminel de Montréal - Cité par A. Fauteux, BRH (1917), 338.

43 AN. Colonies. Corr. gén. C 11, vol. 51, fol. 275.

44 Ibid., D 2 C, 47, fol. 434. - Voir aussi: 4 avril 1730. Le Ministre à Beauharnois, Ibid., série B, vol. 54 , fol. 425 .

4522 mai 1731. Le Ministre à M. Philbert. AN. Colonies. Série B, vol. 55, fol. 537; et 8 avril 1732 . Le même au même, Ibid., vol. 57, fol. 622 . 
Veut et ordonne qu'arrivant son décès, son corps soit enterré en l'église de cette paroisse, et y être inhumé, suivant que son exécuteur testamentaire le jugera convenable, auquel il s'en réfère pour ce entièrement, même pour sa sépulture, le cas échéant qu'elle ne se puisse faire en ladite église cathédrale de Québec.

Veut et ordonne que sur les biens qu'il délaissera au jour de son décès, il soit prélevé la somme de cent livres pour être employées en messes basses de Requiem à son intention et pour le repos de son âme...

Veut qu'il soit aumôné aux pauvres honteux de la paroisse la somme de vingt livres...

Quant aux restes de tous ses biens, tant en meubles qu'immeubles à lui revenant, tant à cause du décès de ses père et mère qu'autres parents desquels il a le droit d'être héritier, et notamment ceux (les biens) qu'il a actuellement en l'ancienne France..., le testateur les donne et lègue aux sieurs De Varenne, La Verandrie, veuves Livilliers et Silvain, ses cousins et cousines germaines pour en jouir en toute propriété...., auxquels susnommés ledit testateur en fait don et legs, (et) au défaut de l'un d'eux, aux survivants et à leurs enfants.

Pour tous lesquels biens connaître et posséder, déclare ledit testateur qu'il a laissé en France entre les mains du sieur Gaignier, entrepreneur des ouvrages du Roy en la ville de Saumur, son procureur, une procuration et compte des biens qu'il pouvait avoir auxdits lieux ou aux environs, aux fins de les vendre, ne l'ayant pu faire à un prix avantageux, lequel sieur Gaignier donnera toutes connaissances de la consistance desdits biens.

Déclare ledit testateur qu'il a remis pour lui conserver dans le vaisseau au sieur Galocheau un sac dans lequel il y a quelques espèces, qu'il le prie de remettre audit sieur exécuteur testamentaire, aux fins d'être employé et remis au désir du susdit testament, - pour lequel exécuter ledit testateur a nommé et choisi la personne de Messire Plante, prêtre, chanoine de cette cathédrale... se dessaisissant en ses mains de tous ses biens, suivant la coutume.

Ainsi fait, dicté et nommé par ledit testateur audit notaire, (en) présence des témoins ci-après nommés ...: sieurs Pierre-François Chalou, maître boulanger, et Charles Prieur, maître perruquier, demeurant en cette ville, rue du Saut-au-Matelot, l'an 1734, le 3e jour de septembre..., et a ledit testateur déclaré qu'il savait signer mais qu'il ne le pouvait..., attendu la faiblesse dans laquelle il se trouvait actuellement.

l'ordonnance.

Et ont les susdits témoins signé avec nous, notaire, lecture faite suivant

(Signé:) Prieur - Chaslou - Barolet, notaire royal.

Comme on le voit, la succession de Charles Philibert se montait à quelques effets personnels et à quelques propriétés en France. Le tout ne semble pas avoir eu beaucoup d'importance et on ne trouve aucune trace de cet héritage dans les documents connus concernant les La Vérendrye au Canada.

Ceux-ci, ses cousins germains, semblent avoir été les seuls proches parents du testateur.

Celui-ci, trop malade le 3 pour signer le testament, mourut le lendemain. Il devait être atteint d'une maladie contagieuse, car il fut enseveli le même jour, dans la cathédrale de Québec. 
Pièce No 37.

(Acte de sépulture de Charles-Gabriel Philibert. - Registre des baptêmes, mariages et sépultures de Notre-Dame de Québec, 4 septembre 1734).

Le quatrième septembre 1734 , a été inhumé dans l'église, proche le bénitier, du côté de Notre-Dame-de-Pitié, le corps de Monsieur Charles Philber, enseigne en pied dans les troupes, âgé de soixante ans, décédé le même jour à quatre heures du matin, après avoir reçu les sacrements de l'Eglise et donné des marques de piété. autres.

Furent présents: Monsieur Degli, Noël Levasseur, ecclésiastique, et

Plante, prêtre.

Le vaisseau Le Rubis, qui avait amené Charles-Gabriel Philibert, était parti de La Rochelle le 29 mai et n'était arrivé à Québec que le 16 août, après une traversée de quatre-vingts jours, accomplie dans les conditions les plus pénibles, par une mer démontée. Mais cela fut peu de choses en comparaison des souffrances dues au manque d'hygiène et au spectacle quotidien de la maladie et de la mort, qui régnèrent en maîtresses durant cette interminable traversée, et dont les passagers furent les témoins „ou les victimes.

Le navire avait à son bord Mgr Dosquet, évêque de Québec, trois sulpiciens, quatre ou cinq jessuites, dont le Père Aulneau, qui devait périr victime des Sioux en 1736 , et le Père Nau, autre missionnaire, destiné aux Iroquois du Saut-Saint-Louis, plus de cent soldats et un certain nombre de faux-sauniers, qui avaient passé un an dans les prisons de France.

Les soldats étaient infestés de vermine, qui se communiqua bientôt à tous les passagers. Plusieurs des faux-sauniers étaient presque nus et couverts de plaies purulentes. La contagion se répandit dans tout le navire et la traversée se fit dans des conditions atroces. Malgré le dévouement des missionnaires et de plusieurs passagers, vingt personnes succombèrent.

Arrivé malade, Charles Philibert fut bientôt aux portes de la mort et succomba.

L'admirable Père Aulneau, parmi beaucoup d'autres, se surmena pour secourir les malades et les mourants. Il eut le bonheur de tenir jusqu'au bout, mais, arrivé à Québec, il tomba malade à son tour et fut plusieurs semaines entre la vie et la mort, mais sa constitution robuste eut le dessus et il se remit complètement. Il était réservé à d'autres combats ${ }^{46}$.

46 Lettre du père Nau au Père Richard, à Bordeaux. Québec, 20 octobre 1734. Texte dans RAPQ (1926-1927), 267-269; et autres lettres du même et du Père Aulneau à divers personnages: octobre 1734, dans la même série. 
Telles furent les conditions dans lesquelles Charles-Gabriel Philibert revint au Canada pour y mourir.

Il signait indifféremment: «Philbert» ou «Philibert».

\section{CONCLUSION}

En dehors des documents officiels et des rapports avec la Cour, concernant les découvertes et les grades militaires, - les démarches de La Vérendrye entre 1724 et 1727 et les faits concernant la succession de Charles-Gabriel Philibert en 1734 sont les dernières relations connues de nos Gaultier avec la France.

Antonio Champagne, c.r.i.c., Saint-Boniface, Manitoba.

\section{Articles à paraître :}

Paul-André LECLERC, ptre: Mariage sous le régime français

Jean-Paul WALLOT: La querelle des prisons (1805-1807)

Robert-Lionel SÉGUIN : La Canadienne aux XVII et XVIII siècles

ROBERT-SYLVAIN, e.c.: Séjour mouvementé d'un révolutionnaire italien à Toronto et à Québec

Fernand OUellet: Papineau et la rivalité Québec-Montréal (1820-1840)

MM. GaUcher, M. Delafosse et G. DeBIEN : Les engagés pour le Canada au XVIII ${ }^{e}$ siècle

Thomas MATHEson: La Mennais et l'Education au Bas-Canada

Robert LE BLANT: Le livre de raison de François de Tapie de Monteil, capitaine au régiment de Poitou (1661-1670)

Pierre Massé: Problèmes acadiens en Poitou 